\title{
Index
}

AbuGhraib 5, 37, 62, 75, 89, 91, 157

see also interrogation; Iraqi prisoner abuse scandal; prisoners; torture

academic institutions $6,20,23,105,164$, $172-5,177,184,188$

act of war 5, 31, 38-40, 42-3, 53, 122, $125,156,176$

see also Twin Towers

ACTA see American Council of Trustees and Alumni

Afghanistan 3, 9-16 passim, 36, 43, 50, 58, 73-5, 78-84 passim, 89-90, 93, 109, 118, $123,125,127,130-7$ passim, 141, $146-7,151,155,157-9,162,168$, 170-1, 174, 180, 185, 187

see also Operation Enduring Freedom; Taliban

African embassy bombings 16

al Qaeda 11, 37, 43, 50-8 passim, 75, 97-8, 102, 105, 109, 111, 128-30, 158, $163,172,174-5$

Albright, Madeline 144, 146

Algeria 37, 93

alien $5,61,70-5,90,108$

invasion movies 71

terrorists $71-2,74$

see also enemy aliens; foreign terrorists

Alien Enemy Bureau 71

alternative counter-terrorism strategies 6, 40, $140-1,184,188$

alternative discourses 19-20, 37, 105, 153, 159, 161,168

alternative narratives $27,51,56,58,154,159$, 161,188

alternative readings $31,54-5$

American Council of Trustees and Alumni 175

American exceptionalism 35, 142, 144, 154,186

see also God's chosen nation; indispensable nation; nature's nation

American foreign policy 2-5, 31, 37, 54-6, $76,108,123,158,162-3,167,170$, 186
American hegemony 52, 58

American military 12, 15-16, 37, 43, 47, $55,58,108,135,183,186$

American political discourse 68, 83-4, 154

American political life 2, 20, 84, 114, 122, 142,177

Amnesty International 13, 89

analogies 40-1, 47, 51, 58, 158

Anderson, Benedict 61 see also imagined community

animals 5, 48-9, 73, 75

see also inhuman terrorists

Annan, Secretary General Kofi 12

anti-Americanism 13, 186

anti-globalisation $19,23,55,83$

anti-modern 53, 55-6

Argentina 131, 183-4

assassination 9, 14, 29, 33, 40, 95, 123, $130,146,180,182$

see also covert operations

asylum seekers 13,182

axis of evil 44, 46, 68, 106, 146-7

Baghram 73

see also Afghanistan; Guantanamo Bay

barbarism 2, 5, 21, 31, 38, 41, 47-59 passim, 62, 66, 73, 90, 140, 142, $154-5,160,176-7$

see also new barbarians; savage

Bergen, Peter 56

bifurcation $86,88,184$

bin Laden, Osama 19, 56, 65-6, 129-30, $138,141,144,157,168,170$

see also al Qaeda

binary structure of language $21,48,62-3$, $72,82,146,149$

biological weapons $22,96,101-2,104-7$, $161,173,177$

Blair, Tony 91, 164, 172

Blunkett, David 111

Britain 10, 16, 37, 41, 91-3, 116-18, 131, 163, 180, 183

Bush doctrine 11, 126 
Camp X-Ray 182 see also Guantanamo Bay

cancer 73-5, 90, 92, 112, 119, 168

see also inhuman terrorists

Carlos the Jackal 98, 111

casus belli 125, 130, 141

see also jus ad helium; jus in hello; just war

catastrophic terrorism 103, 105-6

see also super-terrorism

censorship 116, 169-71

CentralAsiall, 74, 125, 127

Central Intelligence Agency 12, 15, 17, $109,111,116-17,161,183,187$

Chechnya $37-8,74,158,185-6$

chemical weapons $22,96,102-7,161$, 173,177

China 13, 49, 113, 131, 124

Christian 48, 66, 68, 77, 122, 142-3, $151-2,175-7$

see also fundamentalist

CIA see Central Intelligence Agency civil liberties 3, 14, 70, 78, 90, 100, 111, $117,160,182$

civil war 24, 118

civilian casualties 9-10, 36-8, 43, 123, $132-7,146,158,170-1,176,183$

civilisation 2, 5, 21-2, 31, 41, 47-62 passim, 73, 78, 95, 99-100, 111, 113-14, 120-1, 124, 135, 140, 142, $151,154-5,157,160,175-7$

civilising mission 49

clash of civilisations 47

clear and present danger 100, 173

Clinton, President Bill 6, 27, 43, 98, 107, $155-6,166,186$

Coalition 10, 12, 59, 62, 89-91, 127-8, $130-2,137,182$

cold war $3,5,8,16,20,44-7,51,57-8$, $65,68,71,79,92,99,109-10,114$, $138,140,150,152,154,160-1$, $170,178-9,184$

collateral damage $83,130,132-5,182$

Colombia 11, 13, 15, 93, 131, 185-6

consensus 1, 16, 124, 160, 175, 181, 183

consenti, 8, 24, 126, 152, 165

convoy of death 89

counter-violence 5-6, 36, 43, 75, 88, $120,139,181,185,188$

covert operations $8-10,18,141$

coward 56-7, 80, 82, 108, 171 crime 5, 14, 40, 63, 89, 104, 117, 119, 139,182

against humanity $31,38,97$

of the century 40,43

criminal $6,10,38-9,40,43,65,108,115,117$, $126,141,149,155,161$

critical discourse analysis 4, 24-7

Croats 24, 36, 118

cruel 5, 42, 48-50, 62, 89, 97, 132, 144

see also barbarism; savage

crusade $67-8,128,142-3$

Cuba

see Guantanamo Bay

cycle of violence $37,43,120,185$

danger $4-6,11,22,24,35,47,53,64,66$, 74, 76, 88, 92-101, 103-16, 118, $120,154,156$

see also risk to personal safety

Davis, Rick 171

decontextualise 43-4, 97, 157

defensive war 5, 118, 125, 148

see also pre-emptive war; preventive war

dehumanise 5, 49, 60-2, 69-70, 73-5, 90-1, 118, 181, 183

delegitimise 116, 157, 183

democracy $1,5,16,45,52,54-5,57-8$, $95,100,120,123,142,184$

Democratic Republic of Congo 3 7-8

Democrats 3, 6, 119, 130, 159, 160, 178

demonise 5, 6, 44, 49, 53, 55, 59, 60-2, $70,82,90-1,144,156,161,181$, 183

demonological move 66, 70

Department of Homeland Security 3 , $14-15,17,72,116,161,187$

devil 66

different war 124, 148, 150-1

see also new war

dirty war $146,182-3$

disease $5,37,63,73-5,92-4,103,112$, 119

see also inhuman terrorists

dissent 2, 6, 20, 69, 113, 116, 120, 168, 183-4

dissident 13, 23, 60, 97, 99, 100, 106-7, 139

divine calling 67, 141

see also God's chosen nation; historic calling; indispensable nation 
Draculic, Slavenka 181

East African embassies bombings 43

'enabling other' 61,76

Enemy Alien Act of 179871

enemy aliens $5,70-2,74$

enemy combatants $3,6,75,156$

enemy 'other' $60-1,69,72,90,181-3$

enemy terrorists $61-2,65,70,74-6,96$, 108,161

enemy within $5,63,71,96,112-14,154,162$, $175,183-4$

enhanced power $3,13-14,19,96,108,113$, $148,162,187$

see also exercise of power; power

epistemic anxiety 30

ethnic cleansing 4, 180-1

European 35, 50, 93, 149

evil

empire 44, 46, 68, 116, 138, 156

terrorists $5,18,46,66-8,80,87,91$,

135

see also axis of evil; Christian; good;

Manichean

evil one $66,68-9,82,101$

evildoers 5, 61, 68-9, 108, 128, 143

exceptional grievance $32,35-7,181$

see also grievance

exercise of power $2-3,19,25,27,99,117$, 178,188

see also enhanced power; power; powerful discourse; will to power

faceless $4,74-5,90-1,94,108$

see also inhuman

Fadlallah, Sheik 183

Falk, Richard 122, 173

fascism 42, 44, 47, 57, 116, 129-30, 139, 148

see also communism; Nazism

Federal Bureau of Investigation 14-15, 17,

$23,116,185,187$

First Gulf War 58, 141 see also Iraq; Saddam; Second Gulf War

Fleischer, Aril 70

Ford, Henry 44

foreign $61,70-2,74,79,113,115,169$, 172

aid programmes 12

enemy $41,61,70-1$ fighters $89-90$

terrorists $71-3,122$

see also alien; enemy, within; foreigner

Foreign Terrorist Tracking Task Force 15, 72

foreigner $60-1,70,72,114-15,184$

Forth Freedom Forum 16

foundations see think-tanks

freedom

fighters 62

of action $39,75,99,126$

see also Operation Enduring Freedom; Operation Iraqi Freedom

Freedom Promotion Act of 200212

fundamentalist 66,122

see also Christian; Islamic

fundamentalism

gendered language $6,79,157-8$

genealogy 27, 47, 71, 132, 155

Geneva Conventions 6, 60, 74, 90, 147,

149, 185

see also international law; laws of war

genocide $23,33,50,151,180-1$

Georgia 11

Germany 44, 131, 158, 167, 183

Gilmore Commission 107

Giuliani, Rudy 78

global violence $4,152,185$

globalisation 5, 19, 31, 41, 47-8, 51-3,

$57-8,158$

God's chosen nation 35, 142, 149, 154

see also American exceptionalism; divine

calling; historic calling; indispensable nation

good Americans 5, 76, 78, 80, 82, 87

good war 78, 121-4, 129, 135-6, 140-1, 146, 149-52, 154, 158, 167, 174, 176

see also evil; just war; virtuous war grammatical construction 55, 84, 86-7 grammatical forms 2, 21, 85 grammatical strategies 27,138 grievance $31,34,36-8,54,60,63,69-70,75$, $80,83,181,183$

see also exceptional grievance; victim-hood ground zero 4, 18, 35, 43-4, 57, 88, 92, 181 see also Hiroshima; Twin Towers; year zero 
Guantanamo Bay 8, 12, 50, 73, 91, 123, 157 see also Camp X-Ray

\section{Haiti 115}

hate $21,49,54-5,58,60,62-4,69-70$, $77-8,81,118,136,140,181$

hegemonic discourse 19, 159

hegemony $20,52,58$

heroes $59,79-82,104,136,157,161$

heroic $2,5,42-3,57,59,62,77,80-2$, $121,161,175$

see also gendered language

Hiroshima 43, 57, 104-5, 121, 181 see also ground zero

historic

calling 146, 151, 160-1, 174

responsibility $141-2,152$

see also divine calling; God's chosen nation; indispensable nation

historical analogy 40, 43, 47, 150

historical narrative $41,45,50,58,68,78$, $88,129,138$

history $7-8,19,22-5,27,32-3,35-6$, $42-4,46,48,50,52,57-8,67,73$, $82,84,87,97,100,104,135$, 139-40, 143-6, 149, 155, 158, 182, 185

Hollywood 30, 56, 82, 103, 167, 170

holy war $142-3,183$

homeland 70, 79, 81-2, 99, 113, 120, 157

defence 15,72

security 8,116

see also Department of Homeland Security; gendered language

Hoover, J. Edgar 71

Hughes, Karen 26

human rights $6,12-13,19,60,70,73,75$, $78,89,117,151,181,183$

violations $13,37,91,98,100$

Hutu 11, 118

hybridity $7,44,144,154$

see also intertextuality

hyper-reality 30

ICC see International Criminal Court

iconic 7, 18, 33, 82, 165

iconicisation 37

identity $5,48,54,58-62,75,77,79,82$, $87-9,91,108,115-16,162,181$ creation 49,83

markers 50, 61, 77

ideologically driven political project 117

ideology 42, 45, 57, 63, 116, 156, 158,

160

imagined community $61,77,87,114-15$, 182

see also Benedict Anderson

immigration 3, 14, 52, 71

Immigration and Naturalisation Service Contingency plan 71

Indiall, 13, 115, 131

indispensable nation 42, 144, 146, see also American exceptionalism; divine calling; God's chosen nation

Indonesia 11, 15, 131

infection 70, 73-4, 112, 114

see also inhuman terrorist

Infinite Justice 67, 142

inhuman terrorists 4-5, 59, 61, 73-6, 89-91, 108, 122, 135, 176-7, 182

see also animals; barbarism; disease; infection

innocent $2,5,31,49,54,76,79,85-6,94$, 97, 103, 133-4, 140, 160-1, 171, 173

Americans 18, 36, 62, 83-5, 154, 171, 176

civilians $6,36,83-4,104,108,121$, 135

victim $34,54,58-9,62,81,83,85$

Institute for American Values 173

instrumental violence 60,93

insurgency $4,10-11,23,156,184$

insurgent 11, 13, 91, 139, 183

intelligence 3-4, 90, 101, 109, 131

agencies $12-13,98,116$

failure $82,98,120$

gathering 11-12, 16, 90, 108, 141

international cooperation $3,8,131,141$

international law 11, 13, 39, 90, 99, $125-6,129,132,146-7,173$

international order $48,99,185$

international terrorism $14,100,111,176$

International Criminal Court 38, 130, 185

interrogation $11-12,14,60,73,75$, 89-90, 135, 149, 182-3

see also Iraqi prisoner abuse scandal; torture 
intertextuality $69,154-5$

see also hybridity

IRA see Irish Republican Army

Iran $11,44,68,108,114$

Iraq $3,8,10-12,15-16,18,22,41,44$, $50,56,59,68,73-5,78,81-3,88$, $90,93,104,108,114,118,123$,

126, 130, 134-49 passim, 155, 158, $160-3,167,170,172,176,180$, $182-3,185,187$

see also First Gulf War; Operation Iraqi

Freedom; Saddam; Second Gulf War Iraqi prisoner abuse scandal 37, 59, 83, 89, 117,182

see also Abu Ghraib; interrogation; torture Irish Republican Army 37, 87, 91, 182-3

Isaacson, Walter 171

Islamic fundamentalism 116

Israel 13, 37-8, 54-6, 58, 64, 93, 158, $182-3,185-6$

Italy $44,131,158,183$

Japan 33, 42, 44, 50, 75, 131

Japanese 42-3, 63, 71, 114, 181

see also Hiroshima

jihad 110, 144, 174, 183

Judas story $63-4$

jusadbellum 124

jus in hello 124

just war 6, 121-2, 124-6, 129-31, 134, $137-41,146-7,152,168,171$,

$173-6,178$

see also casus belli; good war; virtuous war justice $21,37-8,56,59,67,83-4,87$,

123-32 passim, 137, 143-9 passim, $151-2,155,176$

Kashmir 11, 38, 74, 93, 185-6

Kennedy, John F. 29, 33

Kerry, Senator John 3, 160-1

Khobar Towers bombing 43, 84

King, David 93

knowledge 2, 8, 16-19, 21, 25, 57, 102, $133,138,161,165,168,170,178$, 188

Kosovo 123, 132, 141

Kuwait 10, 41

Land, Richard 176 last resort 6, 121, 124, 139-41

see also just war

laws of war 11, 60, 147, 185

see also Geneva Conventions; pre-emptive war

Lebanon 56, 84

legitimacy 39, 129-30, 132, 147-8, 151, 172

Libya 114, 126, 155

Lockerbie bombing 16, 40, 126

Lynch, Private Jessica 79, 157

Madrid train bombings 92-3, 111

Maher, Bill 171

Manichean 154

Manningham-Buller, Eliza 92

McCarthy 14, 20, 114, 175, 184

media 3, 6, 13, 17-26 passim, 30, 59, 71, 93-5, 111, 119, 123, 131, 135, 153-72 passim, 177-8, 181, 184, 188 frame 20,165

mediaocracy 169

memory $33,40,43,82,122-3,161-2$ meta-narratives see barbarism; cold war; globalisation; World War II

metaphor 2, 43, 70-1, 74, 112, 127, 132, 138

MI6 12, 116

Middle East 8, 11-13, 55-6, 58, 72, 78, 99, $107-8,125,131,158,175$

military assistance programmes $9,11,13,15$, 131,186

commissions 14, 40, 160

dimensions of war on terrorism 8-11, $13,15-16$

industrial complex 80, 158

Milosevic, Slobodan 24, 118

Mobilization Against Terrorism Act 14

mimetic 91,182 see also violence

moral 4, 36-7, 66, 69, 73, 78, 84-91

passim, 116, 123-5, 136, 140-6

passim, 173, 175-6, 181, 183-4, 188

community $6,28,64,68,70,181-2$

duty $6,87,145$

majority 122

mathematics 181

panic $108,115-6,118$

purpose $45-6,88,131-2$ 
movies 30, 33, 44, 56, 62-3, 66, 69, 71, $80,82,95,100,103-4,111,127$, 145,167

see also Hollywood; television

Mujahaddin 58, 158

murder 5, 22-3, 31, 38, 40, 49, 57, 62, 68-9, 86, 89, 91-3, 97, 103, 106, $110,126,129,143,149,160,166$, 173,184

Muslim 13, 24, 64-5, 72, 77, 113, 118, 182,185

myths $2,6,19,27,33,35-6,41,43-4,48$, $50,58,71,80,84,86-8,111-12$, $127,134,139-45$ passim, 149, 154, $163,171,176,186$

myths of the nation see American exceptionalism; foundational myths; God's chosen nation; indispensable nation

narratives see barbarism; cold war; globalisation; story; World War II

National Day of Prayer 87, 143

National Endowment for Democracy 12 national identity $2,33,61,81$

national missile defence $117,119,159$,

186

national security state $113,152,184$

National Security Strategy 126, 161

National Strategy for Combating Terrorism 15,161

nation-building 16, 186

nature's nation 35, 44, 142

see also American exceptionalism; foundational myths; God's chosen nation; indispensible nation

nazism 23, 42, 78, 141, 144, 167

new barbarians, the $47-8$

new war 39, 124, 148-51, 166-7

see also different war

New York 30, 32-3, 35, 38, 43, 57-8, 78, 80-1, 88, 114, 135, 150, 166-7

North Korea 44, 114

north-south conflict 51

Northern Ireland 33, 37, 158, 182-3

nuclear weapons 43, 45-7, 96, 99, 103, 105-6, 161, 163, 173, 178, 181, 186

Oklahoma City bombing 16, 33

Omagh bombing 33, 37 ontological hysteria 118

opacity $45,52-3,68,81,117,121,130$, 157

Operation Enduring Freedom 9-10, 79, 81

Operation Iraqi Freedom 10, 79, 81, 137

opinion polls $162-3,168$

other, the 49-50, 59-62, 76-7, 108, $117-18,156,181-3$

see also 'enabling other'; enemy, 'other' othering process 59,91

outlaw regimes 106, 110, 154-6

see also axis of evil; rogue states

Pakistan 11-12, 15, 131

Palestinian 54, 58, 182

Palmer Raids 71, 114

Panama 114

parasite $71,74-5$

see also inhuman terrorists

Pataki, Governor George 81

Patriot Act see USA Patriot Act

patriotism 85-6, 88, 170-1, 175, 184

peaceful nation $31,54-5,78-9$

Pearl Harbor 5, 27, 31, 41-2, 44, 100, $122,127,129,140,160,167,181$ see also World War II

Pentagon 11, 17, 34, 36, 39, 56, 62, 67, $80,83-5,97,116,124,135,167$, 169-71, 187-8

Philippines, the $11,13,15,131,186$

PNAC see Project for the New American Century

political discourse 19-20, 75, 83-4, 94, $139,153-4,159,164-5,181,188$

political violence $1,4,6,23,25,32,55$, 59-60, 73, 75, 118, 139, 180-1, 183, 189

popular culture $3,46-7,66,79-80,86$, 100

post-modernists 83,184

power 6-7, 13-14, 18-20, 22-6, 30, 32-7, 41, 44-7, 51, 60, 63, 69, 72, 75-85 passim, 90, 94, 99, 102, 109, 113, $115,121,131,139,143-6,156$, $158,163,174,185,187$

see also enhanced power; exercise of power

powerful discourse 7, 18-20, 32-9 passim, 46, 49-51, 53, 57, 62, 68, 72-3, 78, 80-91 passim, 96-7, 106, 112-14, 
$118,127-9,131,136,138-9$, 142-54 passim, 158-9, 161-72 passim, 181, 186-7

pre-emptive war 120

preventive detention $13,99,113$

preventive war 126

prisoners $3-4,8,37,50,73-5,88-91$, $123,147,151,180,182,186$

see also Iraqi prisoner abuse scandal; torture

Project for the New American Century 174

propaganda $1,170,178$

of the deed 93

public anxiety $22,94-5,115,117$

public diplomacy $1,8,12,26$

Purple Heart 39, 80, 84-5

reading 29, 31, 54-8, 64, 73-4, 79, 83, 124, 139

Reagan, President Ronald 6, 27, 44, 46, 68-9, 84, 111, 155-6, 164, 170, 183, 186

reality effect $89,94,97,118-19$

red scare 113-14, 154

redemptive violence 127

reflexivity 39, 123-4, 146-9, 156, 186

religious war 64,142

see also good war; holy war; virtuous war resistance $20,177,180-1,183,188$

Rewards for Justice programme 127

rhetorical constructions 7, 31, 66, 100, $136,146,148$

rhetorical resources $31,37,66,76,127$

rhetorical strategies $7,27,66,96,101$, $107,131-2,135,147-9,163,178$

risk to personal safety $6,92-5,119-20$

see also danger

Robertson, Pat 122, 176-7

rogue states 5, 96, 103, 105, 107, 114, $116,151,154-5$

Roosevelt, Franklin 33, 42, 63, 87-8

Russia 11, 13, 93, 131, 163

Rwanda 11, 33, 37, 50, 118, 180

Saddam 16, 22, 41, 107-8, 126, 137, 139, 163

see also Iraq; Second Gulf War

Said, Edward 48

Sanchez, Ilich Ramirez see Carlos the Jackal

Saudi Arabia 43, 56, 108, 183 savage $5,48-50,58,60-2,73,86,90$, $108,121,157$

see also barbarism

scourge $73,75,90,112,155$

see also inhuman terrorists

Second Gulf War 10, 22

see also Iraq; Operation Iraqi Freedom; Saddam

security $11-13,111,116,161,182-3,187$

Serbs 24, 36

solutions to political violence $4,6,25,139$, 188

Soviet Union 16, 46-7, 68, 99, 156

Spain 131, 158, 183, 185

spectacle $30,93-4$

Sri Lanka 37

Sterling, Claire 111

story $2,17,31,34-5,41,51,58-9,63$, 78-9, 81-2, 88, 111, 127, 154-5, $158,167,169-70$

Straw, Jack 111

Sudan 43, 155

suicide 5,92

bombings $10,56,183$

see also risk to personal safety

super-terrorism 103, 105

see also catastrophic terrorism

supreme emergency 96, 98-100, 113, 118

symbol 18-19, 51-4, 56-7, 61, 102, 113, $136,138,142$

symbolic 17, 26, 36, 38-9, 43, 48, 80, 85, $93,100,102,142,163,167-8$

generalisation 181

violence 38,100

symbolism 129, 142-3

Syria 11

taboo $22,76,94$

Taliban 9-10, 36, 50, 53, 74, 84, 100, $127,134-6,141,158,171,174-6$

television $9,13,17,29-31,33,36,38,40$, $44,66,71,76,79-80,90,95,111$, $122,127,165-6,169,171,174$

Tenet, George 12

terror state 23,140

Terrorism Information and Prevention System Program (TIPS) 15, 113

terrorist violence 4, 93-6, 98, 137, 187-8 
text 7, 17-19, 24-7, 37, 78, 101, 113, $127,131-2,134,136,138,140$, $144,147-8$

textual analysis 24,25

Thatcher, Margaret 87

theo-politics 122

think-tanks 164, 172, 174, 177

torture $6,11-12,49,74-5,78,89-90$, $130,146,149,151,180-4$

see also Abu Ghraib; interrogation; Iraqi prisoner abuse scandal

tragedy $31-8,82,88,96,143$

see also grievance

treacherous $31,42,44-5,62-3,65,87$, 129,181

treachery $42-3,61,63-4,140$

Truman, President 75, 116, 170

Twin Towers 30, 43, 52, 83, 92 see also World Trade Center

Turkey 11

Unabomber 111

United Nations 12, 37, 89, 93, 125-6

unity $2,6,35,61,77,81,85-8,115-16$, 120,170

unlawful combatants 74, 149

US Commission on International Religious

Freedom 176

USA Patriot Act 3, 14, 17, 159, 161, 170

USS Cole 43

Uzbekistan 11, 131

victim-hood $31,36-8,58,80,83,161$,

181, 183

see also grievance; tragedy

victims $31-2,34-9,43,54,58-9,62$, 79-85, 88, 114, 135-6, 142, 157,

$165,167,171,181-2$

victory $42,46-7,137-9,146,157,161$, 175

Vietnam

syndrome 86

War 1, 47, 86, 100, 122, 135

violence see cycle of violence; global violence; instrumental violence; mimetic; political violence; redemptive violence; symbolic violence; terrorist violence; virtual violence

virtual violence 31 virtuous war 130, 151

see also good war; just war

void of meaning 29-31

war

of 181241,71

crime $11,89,182-3$

on drugs $47,94,154$

see also defensive war; different war; dirty war; good war; holy war; just war; new war; preventive war; religious war; virtuous war; winnable war

Washington $32-3,41,53,58,80,84,88,135$, $142,167,170-1,174,187$

way of life 1, 5, 45, 47, 52-3, 95, 99-100, $112,114,118,149,168$

weapons

of mass destruction 2, 5, 15, 22, 92, 96, 101, $103-8,112,114,140,160-1$

of mass murder 96

see also biological weapons; chemical weapons; nuclear weapons

western

culture 51,85

psyche 48,132

security 92,111

societies $6,51,76,94,181$

will to power 42

see also Nazism

winnable war $6,46,137,177$

WMD see weapons of mass destruction

World Trade Center 32-3, 37, 45, 52, 54, 56, 96-7, 100, 104-5, 156, 162-3, 187 see also Twin Towers

World War I 121, 123

World War II 5-6, 31, 36, 41-5, 47, 57-8, $68,78,83,117,121-3,129,139$, $140-1,144,154,160,167-8,173-4$

see also nazism; Pearl Harbor

WTC see World Trade Center

year zero 43,158

see also ground zero

Yemen 10, 43

Yugoslavia 24, 36, 50

Zimbabwe 13 